\title{
Treating Depression with the Mephenesin Analog Skeletal Muscle Relaxant Methocarbamol
}

\section{Toshihiko Numazawa}

2-1, Ooji, Kusatsu City, Japan

Email: numanlabo@yahoo.co.jp

How to cite this paper: Numazawa, T. (2016). Treating Depression with the Mephenesin Analog Skeletal Muscle Relaxant Methocarbamol. Open Journal of Depression, 5, 40-47.

http://dx.doi.org/10.4236/ojd.2016.54005

Received: October 6, 2016

Accepted: November 19, 2016

Published: November 22, 2016

Copyright $\odot 2016$ by author and Scientific Research Publishing Inc.

This work is licensed under the Creative Commons Attribution International License (CC BY 4.0).

http://creativecommons.org/licenses/by/4.0/

\begin{abstract}
It is difficult to treat depression because there is no established method for treatment. Many drugs have been developed by Pharmaceutical companies to treat depression. Depression affects many patients. It was necessary to focus on "meprobamate" which was once said to be a magic drug. It was found that "methocarbamol" is more suitable for treating depression than "meprobamate".
\end{abstract}

\section{Keywords}

Depression, Methocarbamol, Meprobamate

\section{Introduction}

Despite treatments, such as medication and cognitive therapy, the current major problems with depression are:

1) patients go into remission but are not completely cured,

2) patients quickly relapse after returning to work, and

3) patients have to live with the fact that they have depression.

An effective solution for these issues remains to be established.

To determine a solution that combined a therapeutic drug and method for treating depression, we focused on meprobamate, which is considered the most effective psychiatric drug among all psychotropic agents to date.

We found that complete recovery from depression was possible with methocarbamol, a member of the meprobamate family.

\section{History and Effects of Meprobamate}

Berger, who realized the palliative effects of the antimicrobial mephenesin, worked on developing a drug that could be used in everyday clinical settings. In 1951, his appren- 
tice Ludwig was successful in synthesizing meprobamate, which became the first antianxiety drug. The drug was approved for use in the United States in 1955 and was introduced in Japan in 1956, later becoming popular worldwide as a magic drug (Yamauchi, Kojima, Kurachi, \& Kashima, 2011).

Meprobamate relaxes tense nerves, and thus, was considered to be effective for inducing daytime sleep in night workers and alleviating intellectual neurosis. In addition to Atraxin, sold by Daiich Pharmaceuticals, approximately 20 different forms of this meprobamate, including Erina and Harmonin, have been in the market.

Atraxin, which was the first tranquilizer, was hailed as a new drug that treated neuroses caused by stressors such as noise and complex interpersonal relationships. The drug was targeted at a wide range of people, including male workers, housewives, students taking examinations, and children who cried at night. The drug treated symptoms such as daily irritation, insomnia, shoulder stiffness, erythromania, and nervousness during public speaking. In addition to neuroses and insomnia, Atraxin was presented as the drug that would eliminate unpleasant symptoms such as nervousness and impatience, resulting in a comfortable life.

By the mid-1960s, the prevalence of tranquilizer abuse had become a global problem. The view of the United Nations Commission on Narcotic Drugs was that the failure of governments to regulate excessive advertising by pharmaceutical companies and sales without prescriptions had led to this abuse. As a result, the Commission on Narcotic Drugs called for all countries' governments to tighten control and regulate sales, such as by prohibiting the sale of tranquilizers without a doctor's prescription, in an effort to stop this abuse. In February 1971, Japan also joined the "Convention on Psychotropic Substances," designed to encourage international cooperation in preventing the abuse of psychotropic drugs. Meprobamate was subject to regulation under this treaty (Matsueda, 2010).

People who take meprobamate rapidly build up tolerance and exhibit severe withdrawal symptoms. Without knowledge of its mechanism of action, meprobamate was superseded by benzodiazepines and gradually phased out. The next drug to emerge as a replacement for meprobamate was benzodiazepine, which to date remains the leading psychotropic drug (Yamauchi, Kojima, Kurachi, \& Kashima, 2011).

Based on the above, we understand that the mephenesin analog meprobamate was a very good drug for treating symptoms in people of that era. However, the problem of tranquilizer abuse was unfortunately not resolved.

\section{Properties and Effects of Methocarbamol}

Centrally acting muscle relaxants are drugs that inhibit skeletal muscle tone and involuntary movement; they consist of the following two drug groups.

1) Centrally acting skeletal muscle relaxants: These drugs selectively act on the central nervous system that governs skeletal muscle tone and are used to control skeletal muscle spasms and treat tonic spasms or are used during surgical procedures.

2) Antiparkinson drugs: These drugs have central anticholinergic effects and other 
pharmacodynamic effects and are mainly used for treating Parkinson's disease, which is estimated to affect over one million patients only in the United States (Miyano, Watanabe, \& Sumoto, 2000).

The majority of centrally-acting skeletal muscle relaxants, to which methocarbamol belongs, exerts various effects besides relaxing skeletal muscles and are used to treat various symptoms. For example, some skeletal muscle relaxants are effective as hypnotics and sedatives, whereas others are effective against anxiety and nervousness. While temporary, common side effects include drowsiness, vomiting, abdominal pain, and heartburn. Patients who receive these drugs tend to have dulled motor ability, pitch, and attentiveness (Miyano, Watanabe, \& Sumoto, 2000).

Furthermore, methocarbamol is a carbamate derivative of guaifenesin, a derivative of propanediol (Nagatomo, Kunitomo, Hagiwara, \& Takeda, 2008).

Hence, it combines the characteristics of the therapeutic effect of meprobamate through mephenesin.

As hypothesis, a likely cause of depression is tensed muscles and regions that make contact with the interior and exterior parts of the skull bone (Numazawa, 2016). Methocarbamol is considered to have a therapeutic effect on these areas.

Therefore, the centrally acting skeletal muscle relaxant methocarbamol is also considered to be effective for treating depression.

\section{Dependency and Abuse}

There is a list of psychotropic drugs (Japan) that are recognized to cause dependency, have a potential for abuse, and exert adverse effects in the event of abuse (Nakamura, Sirogami, \& Kimura, 2009).

In the list, benzodiazepines are classified as schedule III drugs. Thus, caution must be paid to dosages and dosing periods of anti-anxiety drugs that contain benzodiazepines. Meprobamate is one such drug and is currently prohibited from use in Japan.

However, methocarbamol is not an anti-anxiety drug; it is a skeletal muscle relaxant.

Therefore, it is not included in the list of psychotropic drugs in Japan and requires no caution with regard to dependency, abuse, withdrawal symptoms, or abstinence symptoms.

As a result, methocarbamol can continue to be ingested at doses and for durations that are necessary and sufficient for treatment.

\section{Association of Methocarbamol with Serotonin/Catecholamine}

Because the amounts of 5-hydroxyindoleacetic acid (5-HIAA) and vanillylmandelic acid (VMA) excreted in urine increase following the oral intake of methocarbamol, results of any clinical urine tests for carcinoid tumors or pheochromocytoma, which measure the drug levels, can produce false positives (Campbell, Sherbaniuk, \& Rigby, 1964).

Two readings are made in a spectrophotometer:

one at the azo-VMA peak at $550 \mathrm{~m} \mu$ and the other at the minimum at $450 \mathrm{~m} \mu$. 
Results are expressed as a ratio: $\mathrm{R}=$ absorbance at $450 \mathrm{~m} \mu /$ absorbance at $550 \mathrm{~m} \mu$.

Any $\mathrm{R}$ value less than 1.2 reflects abnormal concentration of VMA.

On $4 \mathrm{gm}$. per day of methocarbamol, normal $\mathrm{R}$ values were found in the first $24 \mathrm{hr}$. The next 24-hr. specimen gave a purple reaction, and on the fourth day a strong purple was produced that extracted into the amyl alcohol to give an $\mathrm{R}$ value of 0.83 .

5-HIAA is excreted in urine as a serotonin metabolite, whereas VMA is excreted catecholamine metabolite.

In addition, noradrenaline has two metabolites, 3-methoxy-4-hydroxyphenylglycol and VMA. VMA levels are reported to decrease in the cerebrospinal fluid of patients with depression (Takahashi \& Takahashi, 1984).

Structural formulas of Methocarbamol and VMA are presented for comparison (see Figure 1 \& Figure 2; KEGG DRUG).

These structural formulas suggest that the oral intake of methocarbamol increases serotonin and catecholamine-like substances in the cranium.

\section{Confirming the Efficacy of Methocarbamol in Humans}

We confirmed the efficacy of methocarbamol in individuals with depression.

$<$ Conditions $>$

1) Targeted individuals with depression for at least 2 years.

2) Receiving methocarbamol.

3) Divide administration into three times/day, continuing for 1 week.

4) Take the drug at home.

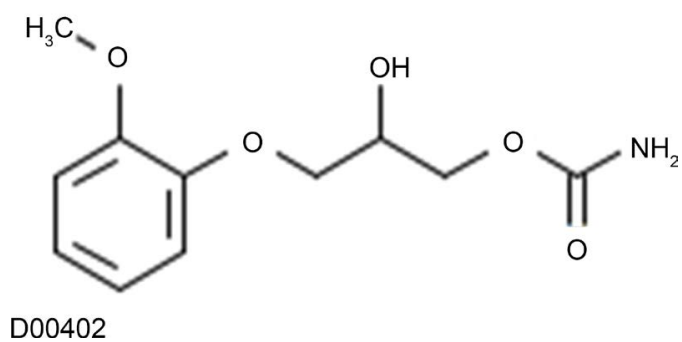

Figure 1. Structural formula of Methocarbamol.

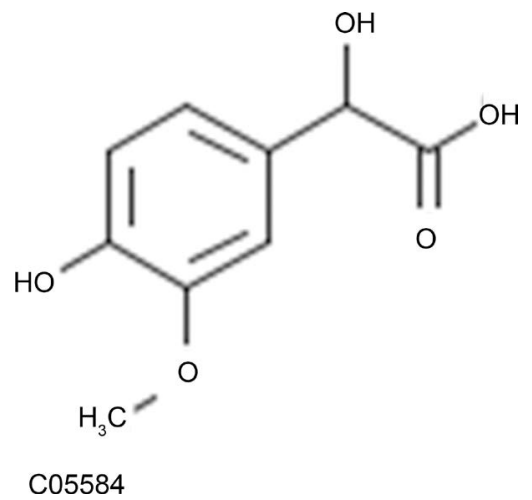

Figure 2. Structural formula of VMA. 
5) Administration set at $3000-4500 \mathrm{mg} /$ day on the first day and then increased to $6000 \mathrm{mg} /$ day based on drug efficacy.

6) Record the daily administered amount.

7) After 1 week of administration, evaluate whether there was an improvement effect (for train of thought and action circuit) on depression.

8) Use a binomial test to discern significant differences between two related groups.

9) Other drugs being concurrently administered may continue to be unchanged.

10) Refrain from drinking alcohol.

$<$ Results $>$

After 1 week of treatment, efficacy was evaluated (for train of thought and action circuit) (see Table 1).

$<$ Discussion $>$

To determine whether methocarbamol was effective, we compared the conditions of the patients before and after administration and performed a binomial test between the two related groups.

As a result, because efficacy was observed in all five individuals, we concluded that the risk rate of $5 \%$ was significant.

Hence, we can suggest that methocarbamol is effective for depression.

This suggests that "methocarbamol" is effective for treating depression.

However, the presence of a therapeutic effect does not mean that depression is completely cured in1week.

\section{Confirming the Efficacy of Methocarbamol in Mice}

A validation experiment of the efficacy of methocarbamol was conducted in mice.

The benzodiazepine analog (thienodiazepine analog) etizolam was used for comparison.

$<$ Conditions $>$

The mice were subjected to a forced swim test.

The experimental protocol is described in 8 .

Comparative verification was performed in the following three groups:

- Vehicle control group (untreated).

- Etizolam-treated group (reagent E-1).

- Methocarbamol-treated group (reagent M-1).

$<$ Dosages $>$

It was determined dosage for mice for experiments for each drug (see Table 2).

$<$ Results $>$

Table 1. Number of patients for test.

\begin{tabular}{cc}
\hline Number of patients & 5 \\
\hline Efficacy observed & 5 \\
No efficacy observed & 0 \\
\hline
\end{tabular}


Table 2. Dosages of drugs for mice.

\begin{tabular}{cccccc}
\hline Reagent & Dosage & Ingredient & Ingredient dosage & Human equivalent dose & Ratio \\
\hline E-1 & $3.3 \mathrm{mg} / \mathrm{kg}$ & Etizolam & $0.033 \mathrm{mg} / \mathrm{kg}$ & $1 \mathrm{mg} / \mathrm{day}$ & 1 \\
$\mathrm{M}-1$ & $260 \mathrm{mg} / \mathrm{kg}$ & Methocarbamol & $200 \mathrm{mg} / \mathrm{kg}$ & $6 \mathrm{~g} /$ day & 6000 \\
\hline
\end{tabular}

Comparison of the efficacy of etizolam and methocarbamol:

The mean durations of immobility of mice in the vehicle control group and two test substance groups were compared and subjected to a test of significance (see Table 3 ).

$<$ Discussion $>$

We found that both reagents M-1 and E-1 exerted a significant effect on depression compared with the control (untreated group). No significant difference was found between treatments with reagents M-1 and E-1 (see Table $4 \&$ Figure 3).

Methocarbamol, a mephenesin analog-like meprobamate, can be considered to have a similar effect as etizolam, a benzodiazepine analog (described in section 2).

\section{Details of the Forced Swim Test in Mice}

The duration of immobility when the mouse was forced to swim inside a cylindrical glass vessel was used as an index to evaluate the antidepressant effect of the test drug(s). The forced swim test is a common model used for conducting experiments on depression (Porsolt, Bertin, \& Jalfre, 1977).

Information on animal subjects:

Species: Mouse.

Family: Slc: ICR.

Gender: Male.

Number of mice: 32 (including 2 spare mice).

Provided by: Japan SLC, Inc.

Microbiological grade: SPF.

Age in weeks during receipt of consignment: 8 weeks.

Age in weeks during trial initiation: 9 weeks.

Individual identification: A label with the test number, cage number, and individual identification number was attached to the cage. A marker was used to record the identification number on the tail of the mice.

Details on Administration:

Administration route: Forced oral administration.

Rationale for adopting the administration route: As per the clinically predetermined administration route.

Administration dose: $10 \mathrm{~mL} / \mathrm{kg}$ by mouse weight.

Rationale for adopting the administration mode: A commonly used method that is most accurate and simple.

Administration frequency: Administered once at $1 \mathrm{~h}$ before the forced swim test.

Pool for forced swim test:

A cylindrical glass vessel with a height of $30 \mathrm{~cm}$ and inner diameter of $16 \mathrm{~cm}$ was 
Table 3. Data of immobility time of mice.

\begin{tabular}{ccc}
\hline Treatment group & Mean duration of immobility $(\mathrm{s})$ & Standard deviation \\
\hline Vehicle control group & 230.8 & 17.0 \\
Reagent E-1 group & 107.3 & 18.5 \\
Reagent M-1 group & 157.7 & 20.1 \\
\hline
\end{tabular}

Table 4. Data of significant difference test.

\begin{tabular}{|c|c|c|c|}
\hline & Study group & $p$ value & Significant difference (5\%) \\
\hline 1 & $\begin{array}{l}\text { Vehicle control group vs. reagent E-1 group (3.3 } \\
\qquad \mathrm{mg} / \mathrm{kg})\end{array}$ & 0.001 & Present \\
\hline 2 & $\begin{array}{l}\text { Vehicle control group vs. reagent M-1 group (260 } \\
\qquad \mathrm{mg} / \mathrm{kg})\end{array}$ & 0.012 & Present \\
\hline 3 & $\begin{array}{l}\text { Reagent E-1group (3.3 mg/kg) vs. reagent M-1 group } \\
\qquad(260 \mathrm{~g} / \mathrm{kg})\end{array}$ & 0.081 & Absent \\
\hline
\end{tabular}

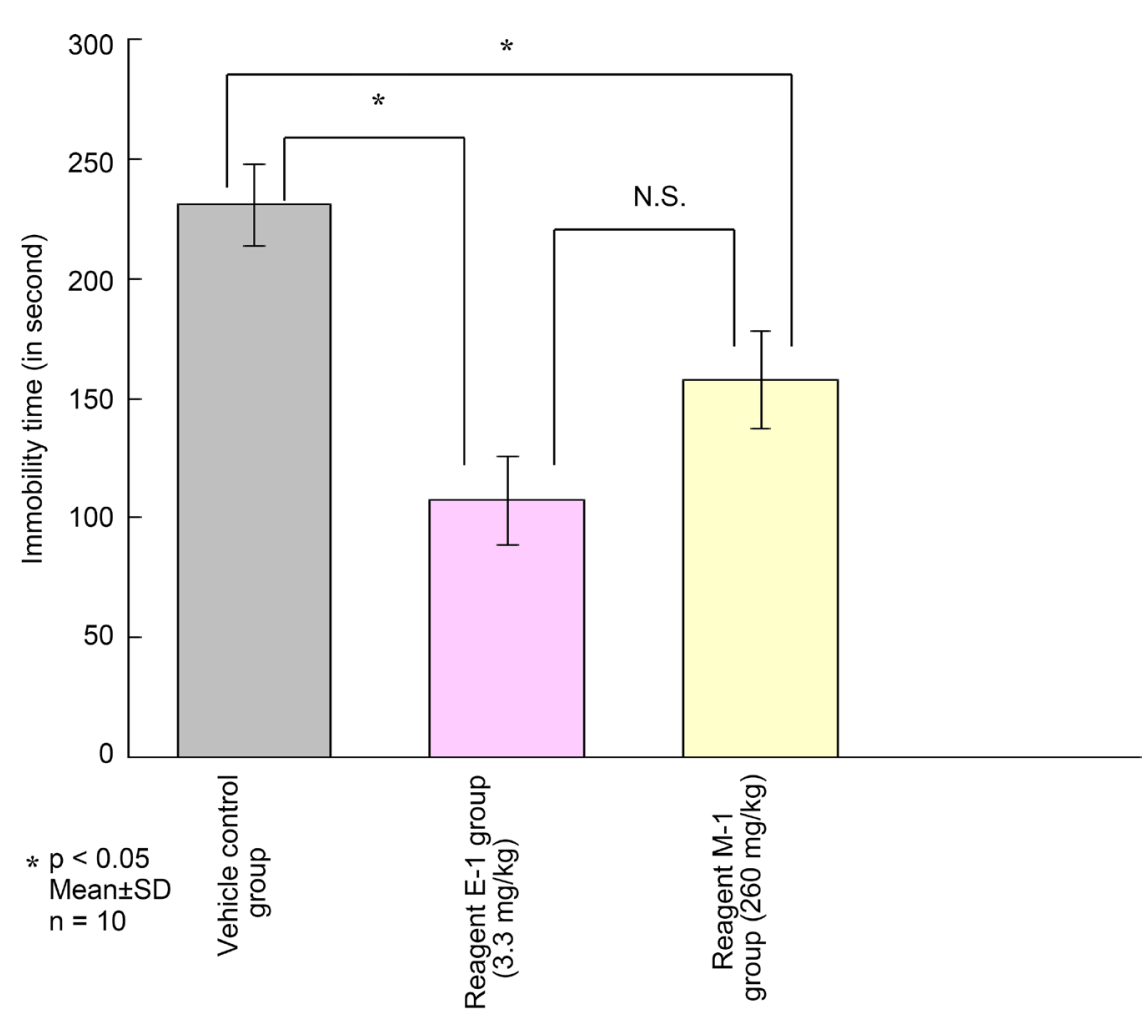

Figure 3. Effectiveness of the test drugs on the duration of immobility of mice during the forced swim test.

used as the pool for the test. The vessel was filled with water until the water level was 20 -cm high. The water temperature was set to $25^{\circ} \mathrm{C}$.

Measurement of the duration of immobility:

The mouse was left in the pool for $10 \mathrm{~min}$. The first $3 \mathrm{~min}$ were considered as the habituation time. The duration of immobility during the remaining 7 min was calculated. Immobility is defined as the state in which the mouse ceases to move both the fore and 
hind limbs and floats only with its head above the water surface (state of immobility). The total duration of this state was recorded as the duration of immobility.

\section{Conclusion}

\section{Based on the above sections $2-7$, we conclude that methocarbamol is effective for} treating depression.

1) Methocarbamol is a mephenesin analog similar to the former drug meprobamate.

2) Centrally acting skeletal muscle relaxants are effective at alleviating tensed muscles and nerves on the inner and outer surfaces of the cranium.

3) There is no dependency; therefore, methocarbamol can be taken in doses and for durations that are necessary and sufficient for treatment.

4) Increases in serotonin and catecholamine levels are likely to be observed in the cranium.

5) It is effective when ingested by humans.

6) Based on experiments, it is effective in mice.

Chlorphenesin carbamate, another mephenesin analog, could also be similarly effective.

\section{References}

Campbell, D. J., Sherbaniuk, R., \& Rigby, J. (1964). False Positive Reaction due to Methocarbamol in the Screening Test for Vanilmandelic Acid (VMA). Clinical Chemistry, 10, 447-450.

KEGG DRUG Database Kanehisa Laboratories. http://www.genome.jp/kegg/drug

Matsueda, A. (2010). Epidemic of Tranquilizers. Core Ethics, 6, 387, 390-395.

Miyano, S., Watanabe, K., \& Sumoto, K. (2000). Medicinal Chemistry Structure-Activity Relationship of Clinical Pharmacy and Medicine [I]. Hirokawa Shoten, 147.

Nagatomo, T., Kunitomo, M., Hagiwara, M., \& Takeda, H. (2008). Medical Pharmacy Latest Pharmacology (Ver. 9). Hirokawa Shoten, 117.

Nakamura, T., Sirogami, M., \& Kimura, K. (2009). Regulatory Laws and System Manual(Ver. 9). Nanzandou, 98.

Numazawa, T. (2016). Principle of and Medical Treatment for Depression. Science Metters. https://www.sciencematters.io/articles/201609000003

Porsolt, R. D., Bertin, A., \& Jalfre, M. (1977). Behavioral Despair in Mice: A Primary Screening Test for Antidepressants. Archives Internationales de Pharmacodynamie et de Therapie, 229, 327-336.

Takahashi, S., \& Takahashi, K. (1984). Pharmacology and Biochemistry of Psychiatric Disorders (Japanese Ver., A. Richard Green, \& D. W. Costain (Translator)). Nishimura Shoten, 80.

Yamauchi, T., Kojima, T., Kurachi, M., \& Kashima, H. (2011). Psychiatry of People Aimed at Specialist(Ver. 3). Igaku-Shoin, 610-611. 
Submit or recommend next manuscript to SCIRP and we will provide best service for you:

Accepting pre-submission inquiries through Email, Facebook, LinkedIn, Twitter, etc. A wide selection of journals (inclusive of 9 subjects, more than 200 journals)

Providing 24-hour high-quality service

User-friendly online submission system

Fair and swift peer-review system

Efficient typesetting and proofreading procedure

Display of the result of downloads and visits, as well as the number of cited articles

Maximum dissemination of your research work

Submit your manuscript at: http://papersubmission.scirp.org/

Or contact ojd@scirp.org 